\title{
Donor-Acceptor Polymer Networks from Triarylborane and Thiophene Building Blocks for Photocatalytic Hydrogen Evolution from Aqueous Solution
}

\author{
Matthias G. Trunk, ${ }^{[a, b]}$ Guiping Li, ${ }^{[a, b]}$ David Burmeister, ${ }^{[a, b]}$ Martin Obermeier, ${ }^{[a]}$ Boubacar Tanda \\ Bonkano, ${ }^{[a, c]}$ Matthias Schwalbe, ${ }^{[a]}$ Michael J. Bojdys ${ }^{\star[a, b, d]}$
}

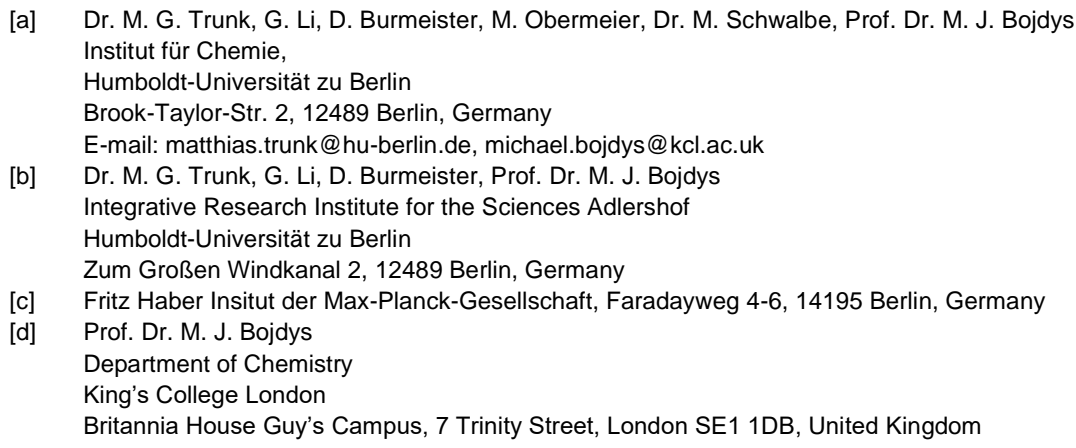

\begin{abstract}
Photocatalytic water-splitting provides a carbon-neutral alternative to energy-intensive electrolysis to store solar energy in the form of hydrogen. Microporous polymer networks are an intriguing platform for the design of increasingly more performant photocatalysts due to their chemical modularity and band-gap tuning potential. Their efficacy depends on the efficient separation of photoexcited electronhole pairs. Conventionally, this is achieved by deposition of expensive platinum as co-catalyst. More recently, however, it was recognized that efficiency of polymer photocatalysts can be improved by incorporation of donor-acceptor motifs into their backbones. While electron donors are plentiful, there is little variety in electron acceptor motifs. We synthesised a series of microporous donor-acceptor networks that contain electron-deficient triarylborane moieties with the unique electronic properties of tricoordinate boron as an electron acceptor. Under sacrificial conditions, these polymers feature hydrogen evolution rates of up to $113.9 \mathrm{mmol} \mathrm{h}^{-1} \mathrm{~g}^{-1}$ that decrease only marginally under omission of platinum co-catalyst. This work outlines a clear synthetic strategy towards truly noble-metal-free photocatalysts.
\end{abstract}

Solar power is the most abundant and accessible energy resource on earth. While hydrogen fuel cells have not (yet) proven sufficiently feasible for mass deployment in passenger cars, they find use in stationary applications. On-site production of hydrogen is gaining importance as it remedies the need for extreme pressurization during transport and storage, which is one of the main hazards associated with hydrogen technology. The only currently available sustainable method to produce hydrogen is via electrolysis, and although the cost efficiency of photovoltaics has risen dramatically over the last two decades, water electrolysis is still an extremely energy-intensive process. The best option to produce hydrogen is by direct conversion of solar energy into hydrogen. In order to become industrially viable and truly sustainable, catalysts need to be stable and composed of earthabundant as well as environmentally benign elements. Those requirements are met by organic photocatalysts such as carbon nitrides and microporous polymer networks. Since carbon nitride was first shown to catalyse the production of hydrogen from aqueous solution, ${ }^{[1]}$ the introduction of concepts such as donor- acceptor interactions, ${ }^{[2-4]}$ increased conjugation between building blocks, ${ }^{[5]}$ and exfoliation to nanosheets ${ }^{[6,7]}$ has given rise to organic polymer networks with remarkable hydrogen production rates under sacrificial conditions of more than $300 \mathrm{mmol} \mathrm{h}^{-1} \mathrm{~g}^{-1} \cdot{ }^{[7]}$ Ideally, a sustainable catalyst should be able to function without added noble-metal co-catalyst, but the majority of current materials loses a significant share of reactivity when used without platinum. Previously, we have shown that organic materials with strong intramolecular donor-acceptor interactions can generally retain high hydrogen evolution activity in the absence of platinum. ${ }^{[2]}$ There, thiophene-based donors and triazine-based acceptors formed donor-acceptor polymer networks that exhibited the highest photocatalytic hydrogen evolution activities in polymer network photocatalysts at the time. In addition, the omission of platinum has a markedly smaller influence on polymer systems with a higher degree of conjugation compared to polymorphic systems in a less ordered state. ${ }^{[5]}$ Recently, Bai et al. utilized computation and automation in an extensive study to reveal several factors contributing to photocatalytic hydrogen evolution activity. ${ }^{[8]}$

Considering the rapid development of the field, the variety of electron-accepting building blocks commonly used is surprisingly small. The triazine $\left(\mathrm{C}_{3} \mathrm{~N}_{3}\right)$ motif is widely used as an electron acceptor in organic photocatalysts due to its simple accessibility via acid-catalysed trimerisation of aromatic nitriles. ${ }^{[2,5,9-12]}$ Aromatic amines can be used to incorporate the larger heptazine $\left(\mathrm{C}_{6} \mathrm{~N}_{7}\right)$ core via nucleophilic substitution to yield $\mathrm{N}$-bridged donoracceptor systems, ${ }^{[13-15]}$ but only the triazine core has been directly cross-coupled via carbon-carbon bond formation to yield highly photoactive materials. ${ }^{[2,16]}$ Recently, sulfone oxides were introduced as electron acceptors for hydrogen evolution photocatalysts. The sulfone oxides serve dual functions as electron acceptors while improving the wettability of the aromatic polymer backbone for enhanced catalyst-substrate interaction. ${ }^{[4,17-20]}$

In this work, we introduce a triarylborane-based electron acceptor for photocatalytic hydrogen evolution. Tricoordinate boron features an intrinsic electron gap, rendering it an excellent electron acceptor. The empty $p$-orbital on boron enables charge- 
transfer interactions with aromatic substituents that give rise to unique optical and electronic effects. Therefore, triarylboranes feature prominently in organic light-emitting diodes and other hybrid materials. ${ }^{[1]}$ Several microporous polymer networks containing triarylborane moieties have been synthesised and their interesting luminescence features were exploited for highly selective chemical sensing and binding of fluoride in the presence of other anions, ${ }^{[22-26]}$ but they have not been studied in the context of photocatalytic hydrogen evolution, yet. Herein, we show that polymer networks based on sulfur-containing donors and boroncontaining acceptors (SBPs) possess high visible-light photocatalytic hydrogen evolution efficacy under sacrificial conditions as well.

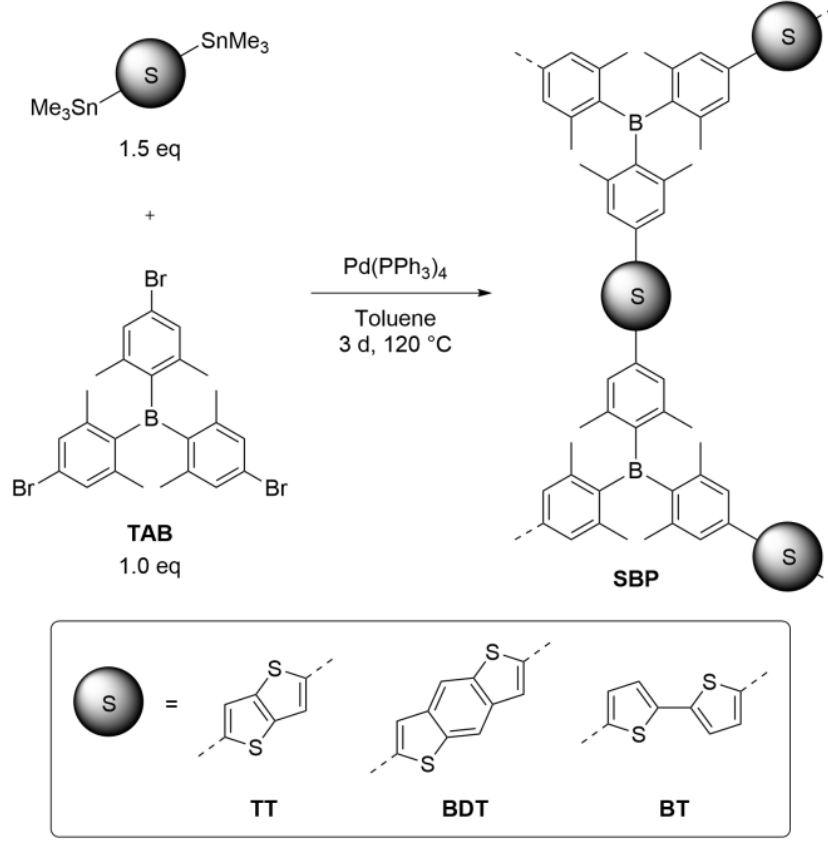

Scheme 1. Synthetic protocol to obtain sulfur-and-boron-based polymer networks (SBPs)

The principal borane building block used herein is accessible via commercial brominated and iodinated 1,3-dimethylbenzene. Selective lithiation of iodide at $-78^{\circ} \mathrm{C}$ followed by nucleophilic attack on boron trifluoride diethyl etherate yields tris(4-bromo-2,6dimethylphenyl)borane (TAB). The stannylated thiophene building blocks are either commercially available or synthetically accessible via a deprotonation-stannylation route. According to our reported procedure, ${ }^{[2]}$ the Stille-type polymerisation of $\mathrm{C}_{2-}$ symmetric donor to $\mathrm{C}_{3}$-symmetric acceptor units in a 3:2 ratio affords powders of TT-, BDT-, and BT-TAB of vibrant yellow and orange colors (Scheme 1).

Low-temperature nitrogen sorption experiments were performed (Figure $1 \mathrm{C}$ and Figure $\mathrm{S} 2$ in the supporting information) and the surface areas determined according to the Brunauer-EmmettTeller (BET) model. TT-TAB and BDT-TAB both feature rigid polymer backbones and large surface areas of 755 and $664 \mathrm{~m}^{2} \mathrm{~g}^{-1}$, respectively. BT-TAB contains bithiophene units with flexible single bonds and consequently exhibits a small surface area of $66 \mathrm{~m}^{2} \mathrm{~g}^{-1}$.
Scanning electron microscopy (SEM) imaging reveals that all SBPs have a cauliflower-shaped morphology typically observed for materials formed via point-nucleation in irreversible coupling reactions, with particle sizes in the range of 50-100 $\mu \mathrm{m}$ (Figures S3-S5). Powder X-ray diffraction (PXRD) shows that these materials are completely amorphous, as expected for products of palladium-catalysed irreversible C-C coupling reactions (Figure S8).

The chemical composition of the materials was determined by elemental analysis, and the observed amounts of carbon, hydrogen and sulfur are in good agreement with the theoretical values (Table S1). The contents of boron, tin and palladium were determined via inductively coupled plasma optical emission spectroscopy (ICP-OES). The amounts of boron range between 1.50 and $1.59 \mathrm{wt} \%$. These are slightly lower than the theoretical values (between 1.90 and $2.04 \mathrm{wt} \%$ ) but are still in reasonable agreement considering the minuscule contribution to the overall composition. Residual amounts of tin originating from unreacted end groups range between 0.01 and $0.03 \mathrm{wt} \%$, corroborating the elemental analysis results and confirming a high coupling efficiency. As is common for polymer networks obtained via palladium-catalysed cross-coupling reactions, small amounts of immobilized palladium were found as well - between 1.1 and $1.5 \mathrm{wt} \% .^{[2,17,27]}$ Detailed ICP-OES results can be found in the supporting information (Table S2).

After combustion at $1000{ }^{\circ} \mathrm{C}$ under oxygen atmosphere a residual mass of around 6 wt\% remains in each case (Figure S7). Taking into account that the weight percentage of boron is slightly lower than expected, this residual mass is in good agreement with the expected mass of $\mathrm{B}_{2} \mathrm{O}_{3}$ - which remains as the only non-volatile oxidation product after carbon, sulfur and hydrogen have been fully oxidised to volatile $\mathrm{CO}_{2}, \mathrm{H}_{2} \mathrm{O}$, and $\mathrm{SO}_{3}$ - plus the residual mass of palladium determined via ICP-OES (Table S6).

Fourier-transform infrared (FTIR) spectra show that the bulk of the signals in the polymer spectra overlap with those of the triarylborane building blocks, confirming their incorporation into the polymer network (Figure 1A). Further signals are assigned to the respective thiophene building block (Table S3-S5). ${ }^{1} \mathrm{H}-{ }^{13} \mathrm{C}$ solid-state cross-polarization magic angle spinning nuclear magnetic resonance $\left({ }^{1} \mathrm{H}-{ }^{13} \mathrm{C}\right.$ CP-MAS NMR) spectra show signals between 108 and 155 ppm assigned to the arylic subunits of the triarylborane as well as thiophene-based building blocks (Figure 1B). Strong additional signals at 21-22 ppm originate from the methyl groups shielding the central boron atom. The ${ }^{11} \mathrm{~B}$ nucleus has a half-integer spin of $3 / 2$ and a quadrupole moment that gives rise to an unusual peak shape in solid-state NMR spectra. All SBPs exhibit one central signal around $69 \mathrm{ppm}$ flanked by a number of central-transition signals that are in excellent agreement with literature (Figure S6). ${ }^{\text {28] }}$

Ultraviolet-visible (UV/Vis) reflectance spectra measured in the solid-state show that the frameworks absorb visible light up to $530 \mathrm{~nm}$ (Figure 2A). Based on the UV/Vis spectra, the direct optical bandgaps of TT-TAB, BDT-TAB, and BT-TAB are 2.58, 2.56 and $2.49 \mathrm{eV}$, respectively (Figure $2 \mathrm{D}$ ). Therefore, all bandgaps are in the range suitable for visible-light photocatalytic proton reduction. ${ }^{[8]}$ 

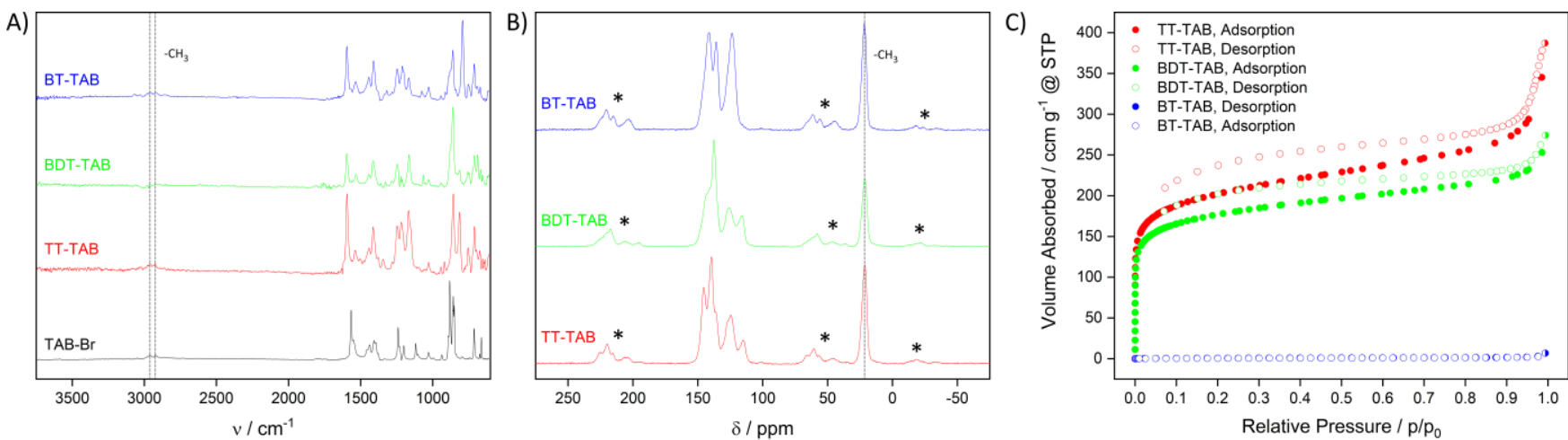

Figure 1. A) Fourier-transform infrared (FTIR) spectra for all SBPs as well as for their principal TAB-Br building block. Dashed lines denote methyl group signals. B) ${ }^{1} \mathrm{H}^{13} \mathrm{C}$ cross-polarization magic-angle spinning (CP-MAS) solid-state NMR spectra for all SBPs, recorded at $8 \mathrm{kHz}$. Asterisks $\left({ }^{*}\right)$ denote spinning sidebands, dashed lines denote methyl group signals. C) Nitrogen sorption isotherms of all SBPs, measured at $77 \mathrm{~K}$.

Photocatalytic hydrogen evolution reactions were carried out using incident light above $395 \mathrm{~nm}$, and the amount of evolved hydrogen was determined by injection of headspace samples into a gas chromatograph equipped with thermal conductivity (TCD) detector. Due to the presence of multiple methyl groups, the synthesised SBPs are hydrophobic. Hence, the catalytic tests were performed in a solvent mixture of equal volumes of water and acetonitrile. To evaluate the catalytic activities, we screened different reaction parameters such as catalyst loading and type of sacrificial agents, and we found that both factors had a strong influence on the catalytic activity. The lowest tested concentration of $1 \mathrm{mg}$ of catalyst per $16 \mathrm{~mL}$ of solvent yielded the highest activity per catalyst mass (Figure S10). Even at such low loadings, we observed a vigorous formation of hydrogen bubbles (see video in the supporting information). It has been noted previously that low catalyst concentrations can result in "severe unevenness and in some cases [...] exceptional reactivity". ${ }^{[29]}$ However, despite the low catalyst loadings tested here, we observe smooth curves and good reproducibility. L-ascorbic acid, triethanolamine, and triethylamine were evaluated as sacrificial electron donors (Figure S11). Similar to other recently reported photocatalysts, L-ascorbic acid yielded the highest activities by far. ${ }^{[3,20]}$ Averaged over five hours, TT-TAB, BDT-TAB, and BT-TAB showed hydrogen evolution rates of $113.9,87.7$, and $74.2 \mathrm{mmol} \mathrm{h}^{-1} \mathrm{~g}^{-1}$, respectively, under addition of $3 \mathrm{wt} \%$ of platinum (Figure 2B). Testing of multiple batches as well as multiple runs on the same batch confirmed the activity of the best-performing material, TT$\mathrm{TAB}$, within a $15 \%$ error margin, as is typically observed for batchbased photocatalytic hydrogen evolution testing (Figure S14). ${ }^{[30]}$ Application of the same conditions to our previously reported sulfur-and-nitrogen-based polymer networks (SNPs) ${ }^{[2,16]}$ showed that for these materials, triethanolamine and triethylamine afford higher activities than L-ascorbic acid (Figure S12). This behaviour seems at first surprising given that acidic media tends to favor proton reduction by enhancing donor-acceptor interactions between protonated triazines and sulfur-containing donors in SNPs. However, following the observation by Yang et al. that ascorbic acid is not a sufficiently strong acid to protonate the triazine nitrogen atoms, we conclude that the intrinsic donoracceptor dyads in SNPs are sufficiently strong for efficient electron-hole separation and high hydrogen evolution activity without the additional protonation effect. ${ }^{[3]}$ Notably, SBPs do not contain any nitrogen atoms that can be protonated, indicating that the intrinsic donor-acceptor interactions in SBPs are equally effective. No hydrogen was detected when experiments were performed without the organic catalyst, water, or sacrificial electron donor.

The photochemical stability of TT-TAB was tested via long-term hydrogen evolution experiments. TT-TAB was irradiated for seven cycles of five hours each with hourly gas chromatography measurements. After each cycle, the initial amount of sacrificial agent was replenished, and the reaction mixture was degassed thoroughly before further irradiation. The initial hydrogen evolution activity decreased continuously by approximately $8 \%$ per five-hour cycle, reaching a residual activity of $43 \%$ after 35 hours (Figure 2F). Assuming total depletion after twelve cycles, the turnover number equals 311657 with respect to triarylborane units, which results in a turnover frequency of $1.44 \mathrm{~s}$ 1.

To trace the decrease in catalytic activity, a sample of BDT-TAB was irradiated for $15 \mathrm{~h}$, recovered, and analysed via FTIR, solidstate NMR, and UV/Vis spectroscopy (Figure S17-S19). In essence, the polymer appears to be chemically and structurally unchanged. These findings eliminate deterioration of the polymer as a likely culprit for a decrease in catalytic activity. As an explanation, we propose that the accumulation of oxidised ascorbic acid in the reaction mixture has a detrimental effect on the catalytic activity. Indeed, when exchanging the solvent after each five-hour cycle, the catalytic activity remained effectively constant after four cycles (Figure S16).

Despite this high activity, the consumed volume of water per cycle ranges on the microliter scale and can be neglected. However, the volume of evolved hydrogen in each five-hour run exceeds $10 \mathrm{~mL}$ compared to the headspace volume of $25 \mathrm{~mL}$. Therefore, we conclude that the calculated amount of evolved hydrogen is likely lower than the actual amount produced due to the leaking of hydrogen. To render the buildup of pressure inside the reactor more tangible, we compared the detected amounts of hydrogen when using two different gas-tight syringes, one equipped with a valve and one without a valve. Normally, the syringe equipped with a valve is loaded with a headspace sample and the valve closed while the needle is still inside the reactor. In the case without a valve, after drawing the headspace sample, the syringe was briefly exposed to ambient air before being sealed with a rubber septum to avoid the escape of hydrogen. Indeed, the values using a syringe without a valve are lower by about $20 \%$, indicating that significant overpressure escapes during the brief exposure to ambient pressure (Figure S15). 

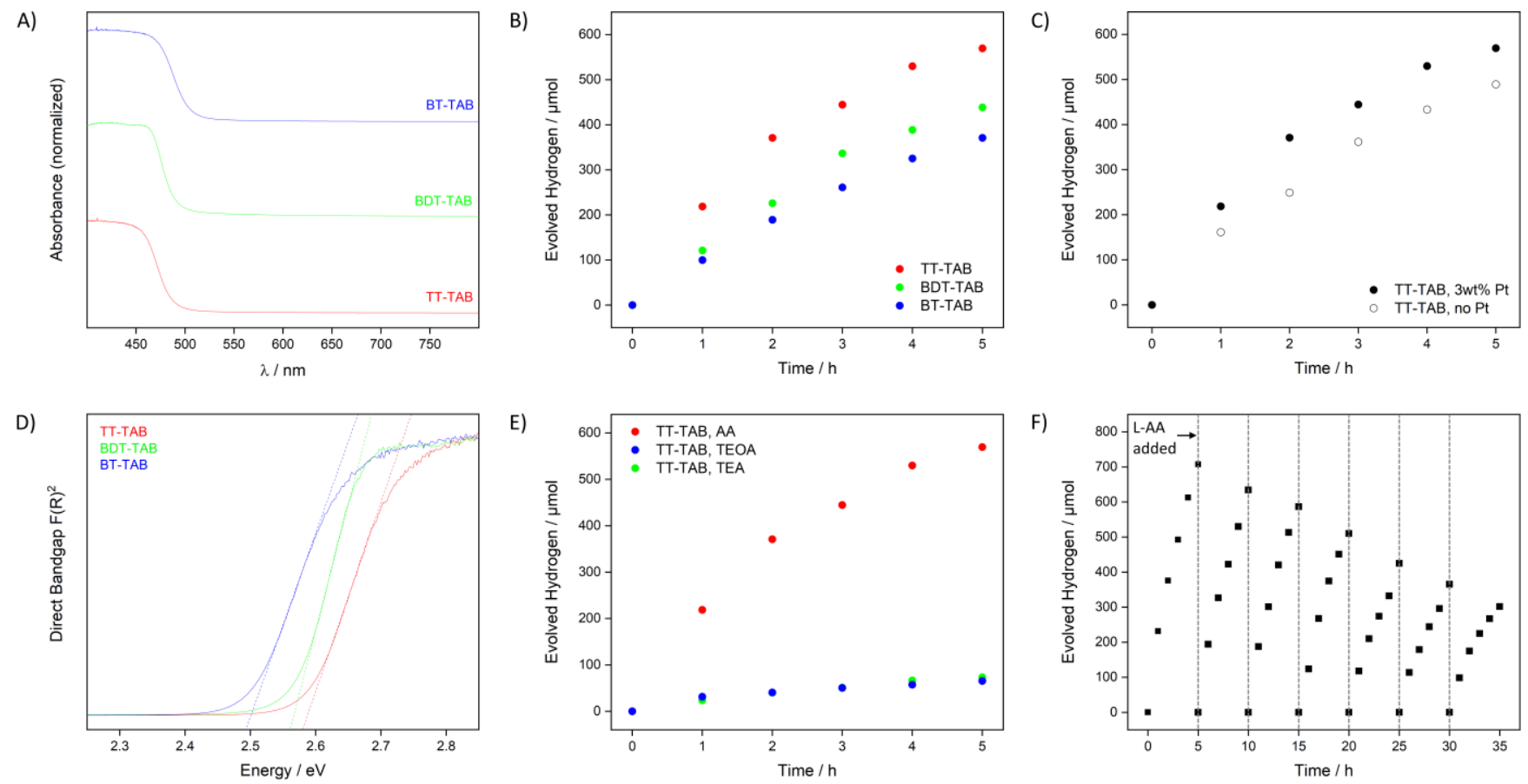

Figure 2. Optical characterization of all SBPs showing A) solid-state UV/vis spectra and D) the derived direct optical bandgaps; B) hydrogen evolution experiments comparing the activity of all SBPs with L-ascorbic acid as sacrificial agent; E) comparison of sacrificial agents using TT-TAB as catalyst; C) influence of platinum as co-catalyst; F) long-term hydrogen evolution using TT-TAB and L-ascorbic acid as sacrificial agent. Dashed lines denote cycle change and addition of fresh sacrificial agent. General conditions for HER experiments using L-ascorbic acid: $1 \mathrm{mg}$ of catalyst, $8 \mathrm{~mL}$ acetonitrile, $8 \mathrm{~mL} \mathrm{~L}$-ascorbic acid (0.1 M), Pt (3 wt\%) unless Pt-free. Further conditions can be found in the SI.

In conventional polymer photocatalysts, omission of platinum as co-catalyst usually leads to significantly lower activity. Recently, Han et al. observed that - also using ascorbic acid as the sacrificial agent - no platinum co-catalyst was necessary to achieve hydrogen evolution rates of $190.7 \mathrm{mmol} \mathrm{h}^{-1} \mathrm{~g}^{-1}$ in an amorphous polymer network. This impressive value amounts to $63 \%$ of the hydrogen evolution activity of the same polymer with added platinum co-catalyst. ${ }^{[20]}$ Other reports show high hydrogen evolution rates using ascorbic acid without platinum as well, but do not provide hydrogen evolution activities with added platinum for comparison. ${ }^{[6]}$ With TT-TAB as the photocatalyst, the omission of platinum leads to a drop in hydrogen evolution rate by only $14 \%$ (from 113.9 to $97.9 \mathrm{mmol} \mathrm{h}^{-1} \mathrm{~g}^{-1}$, Figure $2 \mathrm{C}$ ). At $74.2 \mathrm{mmol} \mathrm{h}^{-1} \mathrm{~g}^{-1}$, the activity of BT-TAB is the lowest in the series, and it is essentially identical with and without platinum (Figure S13).

The sustained hydrogen evolution without platinum co-catalyst is surprising, and we propose that it stems from the electronic makeup of the polymer networks. To determine the lifetime of the excited states, we performed time-correlated single-photon counting (TCSPC) experiments. The lifetimes obtained from triexponential decay fitting are similar to those of previously reported triarylborane-based donor-acceptor systems ${ }^{[23]}$ and other solid donor-acceptor hydrogen evolution catalysts. ${ }^{[3,30]}$ The weighted average lifetime $T_{\text {avg }}$ is longest for BDT-TAB $(0.259 \mathrm{~ns})$, followed by TT-TAB (0.180 ns) and BT-TAB (0.104 ns). Interestingly, the longest lifetime $T_{3}$ has a similar weight in all materials - between 4 and $7 \%$ - and decreases from TT-TAB to $\mathrm{BT}-\mathrm{TAB}$, following the same trend as the observed catalytic activities (Figure S21 and Table S7). This indicates that the first (and possibly second) decay processes are too fast for these excitons to contribute significantly to the catalytic activity, or that additional factors contribute to the activity, such as the total number of generated excitons.

To obtain a qualitative understanding of the donor-acceptor interactions in our polymers, we performed density functional theory (DFT) calculations of the frontier orbitals in a model system that represents a fragment of the polymeric repeats comprising the triarylborane core connected to three thiophene moieties. The obtained Kohn-Sham highest occupied molecular orbital (HOMO) exhibits a node on the boron atom, whereas the lowest unoccupied molecular orbital (LUMO) has a large coefficient on boron, and notably smaller coefficients on the rest of the atoms (Figure S22). From these results we conclude that, after vertical excitation, the excited electron has a high probability density at the boron center. On the basis of these calculations we propose the following mechanism for photoexcitation and hydrogen evolution: (i) photoexcited charges separate and form a boryl radical anion and thiophene radical cation, and (ii) the generated cation is reduced by ascorbic acid. (iii) A second photoexcitation event transfers another electron to the boron center and the emerging electron pair is protonated and forms a borohydride species. Finally, (iv) the borohydride gets protonated and - under elimination of hydrogen $\left(\mathrm{H}_{2}\right)$ - the catalyst regenerates using an electron from ascorbic acid (Figure S23). The exact mechanism will be the subject of future studies.

In summary, we have synthesised a series of highly active hydrogen evolution photocatalysts, and in doing so, we added a readily available electron acceptor to the library of building blocks for visible-light photocatalysts from aqueous solution. Under optimized conditions, the thieno[3,2-b]thiophene-triarylborane copolymer (TT-TAB) produces $113.9 \mathrm{mmol}$ of hydrogen per hour per gram of catalyst. Most importantly, even without added 
platinum cocatalyst, its activity is close to $100 \mathrm{mmol} \mathrm{h}^{-1} \mathrm{~g}^{-1}$, a benchmark value. These findings emphasise the importance of donor-acceptor dyads for effective separation and utilisation of photoexcited charge carriers in efficient photocatalytic processes that do away with scarce noble metals as co-catalysts.

\section{Acknowledgements}

We thank Harald Link and Christina Eichenauer of Berlin Institute of Technology for ICP-OES and TGA measurements, respectively. Thomas Dargel of Humboldt University of Berlin is acknowledged for support performing DFT calculations. M.J.B. thanks the European Research Council (ERC) for funding under the Starting Grant Scheme (BEGMAT-678462) and the Proof of Concept Grant Scheme (LiAnMat-957534).

Keywords: Hydrogen evolution, water-splitting, microporous polymer network, donor-acceptor, triarylborane

\section{References}

[1] X. Wang, K. Maeda, A. Thomas, K. Takanabe, G. Xin, J. M. Carlsson, K. Domen, M. Antonietti, Nat. Mater. 2009, 8, 76-80. Y. S. Kochergin, D. Schwarz, A. Acharjya, A. Ichangi, R. Kulkarni, P. Eliášová, J. Vacek, J. Schmidt, A. Thomas, M. J. Bojdys, Angew. Chemie Int. Ed. 2018, 57, 14188-14192.

[3] J. Yang, A. Acharjya, M.-Y. Ye, J. Rabeah, S. Li, Z. Kochovski, S. Youk, J. Roeser, J. Grüneberg, M. Schwarze, T. Wang, Y. Lu, R. van de Krol, M. Oschatz, R. Schomäcker, P. Saalfrank, A. Thomas, C. Penschke, Angew. Chemie Int. Ed. 2021, anie.202104870.

[4] M. A. Zwijnenburg, Y. Wu, X. Wang, R. S. Sprick, M. A. Little, W.-H Zhu, A. I. Cooper, R. Clowes, L. Chen, S. Y. Chong, Y. Yan, Nat. Chem. 2018, 10, 1180-1189.

[5] D. Schwarz, A. Acharjya, A. Ichangi, Y. S. Kochergin, P. Lyu, M. V. Opanasenko, J. Tarábek, J. Vacek Chocholoušová, J. Vacek, J. Schmidt, J. Čejka, P. Nachtigall, A. Thomas, M. J. Bojdys, ChemSusChem 2019, 12, 194-199.

[6] J. Cheng, Z.-R. Tan, Y.-Q. Xing, Z.-Q. Shen, Y. Zhang, L.-L. Liu, K. Yang, L. Chen, S.-Y. Liu, J. Mater. Chem. A 2021, 9, 5787-5795.

[7] J.-Z. Cheng, L.-L. Liu, G. Liao, Z.-Q. Shen, Z.-R. Tan, Y.-Q. Xing, X.-X. Li, K. Yang, L. Chen, S.-Y. Liu, J. Mater. Chem. A 2020, 8, 5890-5899.

[8] Y. Bai, L. Wilbraham, B. J. Slater, M. A. Zwijnenburg, R. S. Sprick, A. I. Cooper, J. Am. Chem. Soc. 2019, 141, 9063-9071.

[9] D. Schwarz, A. Acharjya, A. Ichangi, P. Lyu, M. V. Opanasenko, F. R. Goßler, T. A. F. König, J. Čejka, P. Nachtigall, A. Thomas, M. J. Bojdys, Chem. - A Eur. J. 2018, 24, 11916-11921.

[10] V. S. Vyas, F. Haase, L. Stegbauer, G. Savasci, F. Podjaski, C. Ochsenfeld, B. V. Lotsch, Nat. Commun. 2015, 6, 8508.

[11] S. Kuecken, A. Acharjya, L. Zhi, M. Schwarze, R. Schomäcker, A. Thomas, Chem. Commun. 2017, 53, 5854-5857.

[12] K. Schwinghammer, S. Hug, M. B. Mesch, J. Senker, B. V. Lotsch, Energy Environ. Sci. 2015, 8, 3345-3353.

[13] K. Kailasam, J. Schmidt, H. Bildirir, G. Zhang, S. Blechert, X. Wang, A. Thomas, Macromol. Rapid Commun. 2013, 34, 1008-1013.
Schwarze, M. Schröder, R. Schomäcker, J. Senker, A. Thomas Energy Technol. 2016, 4, 744-750.

[15] N. Sharma, S. Kumar, V. R. Battula, A. Kumari, A. Giri, A. Patra, K. Kailasam, Chem. - A Eur. J. 2021, chem.202100595.

[16] Y. S. Kochergin, Y. Noda, R. Kulkarni, K. Škodáková, J. Tarábek, J. Schmidt, M. J. Bojdys, Macromolecules 2019, 52, 7696-7703.

[17] R. S. Sprick, B. Bonillo, R. Clowes, P. Guiglion, N. J. Brownbill, B. J. Slater, F. Blanc, M. A. Zwijnenburg, D. J. Adams, A. I. Cooper, Angew. Chemie Int. Ed. 2016, 55, 1792-1796.

[18] Z. Wang, X. Yang, T. Yang, Y. Zhao, F. Wang, Y. Chen, J. H. Zeng, C. Yan, F. Huang, J.-X. Jiang, ACS Catal. 2018, 8, 8590-8596.

[19] A. Vogel, M. Forster, L. Wilbraham, C. L. Smith, A. J. Cowan, M. A. Zwijnenburg, R. S. Sprick, A. I. Cooper, Faraday Discuss. 2019, 215, 84-97.

[20] C. Han, P. Dong, H. Tang, P. Zheng, C. Zhang, F. Wang, F. Huang, J.-X. Jiang, Chem. Sci. 2021, 12, 1796-1802.

[21] G. Turkoglu, M. E. Cinar, T. Ozturk, Molecules 2017, 22, 1522.

[22] X. Liu, Y. Zhang, H. Li, S. A, H. Xia, Y. Mu, RSC Adv. 2013, 3, 21267-21270.

[23] W. Zhao, X. Zhuang, D. Wu, F. Zhang, D. Gehrig, F. Laquai, X. Feng, J. Mater. Chem. A 2013, 1, 13878-13884.

[24] V. M. Suresh, A. Bandyopadhyay, S. Roy, S. K. Pati, T. K. Maji, Chem. - A Eur. J. 2015, 21, 10799-10804

[25] Z. Li, H. Li, H. Xia, X. Ding, X. Luo, X. Liu, Y. Mu, Chem. - A Eur. J. 2015, 21, 17355-17362.

[26] C. Gu, N. Huang, Y. Chen, H. Zhang, S. Zhang, F. Li, Y. Ma, D. Jiang, Angew. Chemie Int. Ed. 2016, 55, 3049-3053. C. Dai, S. Xu, W. Liu, X. Gong, M. Panahandeh-Fard, Z. Liu, D. Zhang, C. Xue, K. P. Loh, B. Liu, Small 2018, 14, 1801839.

[28] P. Király, Magn. Reson. Chem. 2012, 50, 620-626.

[29] E. Jin, Z. Lan, Q. Jiang, K. Geng, G. Li, X. Wang, D. Jiang, Chem 2019, 5, 1632-1647.

[30] B. P. Biswal, H. A. Vignolo-González, T. Banerjee, L. Grunenberg, G. Savasci, K. Gottschling, J. Nuss, C. Ochsenfeld, B. V. Lotsch, J. Am. Chem. Soc. 2019, 141, 11082-11092. 


\section{Entry for the Table of Contents}

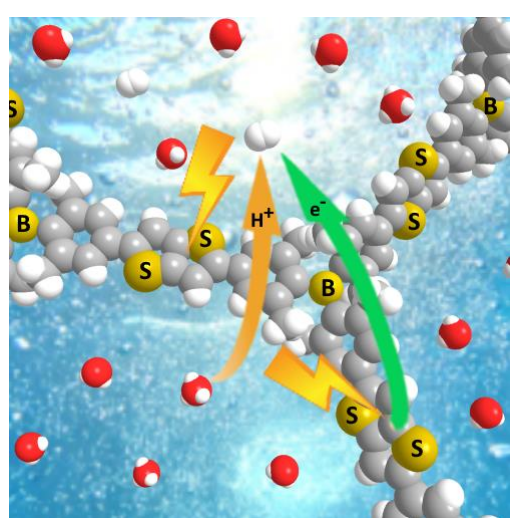

S-lam B-am! We synthesised a series of Sulfur-and-Boron-based polymer networks from strong electron donors and acceptors. The resulting materials separate charges effectively and perform well as hydrogen evolution photocatalysts without addition of noble metals.

Institute and/or researcher Twitter usernames: @TrunkMatthias @bojdysLAB @mjbojdys @HumboldtChem 